\title{
Role of Haematological Score in Early and Rapid Diagnosis of Neonatal Septicaemia
}

\author{
Khan $\mathrm{S}^{\mathrm{a}}$, Baki MA ${ }^{\mathrm{b}}$, Halder $\mathrm{AL}^{\mathrm{c}}$, Haque $\mathrm{A}^{\mathrm{d}}$, Begum $\mathrm{T}^{\mathrm{e}}$, Nahar $\mathrm{N}^{\mathrm{f}}$
}

\begin{abstract}
Background: Septicaemia is well documented as being one of the major causes of neonatal mortality and morbidity worldwide including Bangladesh. A haematological scoring system which includes total WBC count, absolute neutrophil count, platelet count and $C$ reactive protein may be beneficial for the early and rapid diagnosis of neonatal septicaemia. So this study was done to see the role of the haematological score in neonatal septicaemia.

Methods: This observational study was done in SCABU, BIRDEM General Hospital 2 from April 2012 to September 2012. One twenty neonates who were clinically suspected of septicaemia were enrolled in this study. A score of 1 was given for each of the haematological parameters. Blood culture was done in all neonates along with haematological score to prove septicaemia.

Results: In this study 42 neonates had a score of 1, a score of 2 was present in 45 neonates. Score 3 was present in 23 neonates and score 4 was present in 10 patients. Among the 42 neonates having a score of 1, culture was negative in $69 \%$ and $30 \%$ had positive culture reports. In neonates with score 2, it was seen that almost half (46.4\%) had a positive blood culture and the rest (53.3\%) were culture negative. Twenty three neonates had a score of 3 among which $73.9 \%$ were proven sepsis by positive culture reports. Blood culture was positive in $100 \%$ patients with a score of 4 .

Conclusion: The higher the score the greater the chance of neonatal septicaemia. The haematological scoring system may aid the clinicians to use proper antibiotics at the right time and thus reduce neonatal morbidity and mortality.
\end{abstract}

Key words : Neonates, Septicaermia, Haematological Scoring System

(BIRDEM Med J 2017; 7(3): 212-215)

\section{Introduction}

Septicaemia is well documented as being one of the major causes of neonatal mortality and morbidity worldwide. More than one-third of the neonatal deaths

Author Information

a. Dr. Shareen Khan, Registrar, Department of Paediatrics, BIRDEM General Hospital, Dhaka.

b. Dr. MA Baki, Assistant Professor, Department of Paediatrics, BIRDEM General Hospital, Dhaka.

c. Dr. Amrita Lal Halder, Resident Physician, Department of Paediatrics, BIRDEM General Hospital, Dhaka.

d. Dr. Afroza Haque, Consultant, Department of Paediatrics, BIRDEM General Hospital, Dhaka.

e. Prof. Tahmina Begum, Professor of Paediatrics.

f. Prof. Nazmun Nahar, Director General and Professor of Paediatrics, BIRDEM General Hospital, Dhaka.

Address of correspondence: Dr. Shareen Khan, Registrar, Department of Paediatrics, BIRDEM General Hospital, Dhaka, Email: shareenkhan26@gmail.com

Received: November 22, 2016

Accepted: July 31, 2017 are estimated to be due to septicaemia. ${ }^{1}$ In Bangladesh neonatal mortality is $28 / 1000$ live birth. ${ }^{2}$ The most common organisms responsible for neonatal septicaemia are E. Coli, Klebsiella pneumoniae, Staphylococcus aurues, Pseudomonas aeruginosa, Streptococcus saprophyticus, Acinetobacter. ${ }^{3}$ Being a life threatening condition, the rapid diagnosis of neonatal septicaemia is imperative and poses a great challenge worldwide including Bangladesh. Although blood culture is recognised as being the gold standard for the definitive diagnosis of this condition, it is a time consuming and costly procedure and not readily available in rural areas of Bangladesh. This has prompted the evaluation of surrogate markers of inflammation as the possible tools for early diagnosis of bacterial sepsis. Estimation of C Reactive Protein (CRP) levels are potentially useful in this respect. ${ }^{4,5}$ Also haematological parameters 
including platelet count, total leukocyte count (WBC), absolute neutrophil count (ANC) are good predictors of sepsis in newborn. ${ }^{6}$ Neutropenia $<2000-2500 / \mathrm{cmm}$ is believed to be the best predictor of sepsis whereas neutrophilia $>7500-8000 / \mathrm{cmm}$ correlate less well. ${ }^{7}$ Thrombocytopenia has been used as an early but nonspecific marker for sepsis. ${ }^{8,9}$ These investigations are cost effective and reliable and can be used for early and rapid diagnosis of systemic infection in neonates in a short time. So the objective of this study was to observe the role of haematological scoring system in early and rapid diagnosis of neonatal septicaemia.

\section{Methods}

This study was done in Special Care Baby Unit (SCABU), BIRDEM General Hospital 2 from April 2012 to September 2012. One twenty neonates irrespective of gestational age and weight who were admitted in SCABU and clinically suspected of septicaemia (fever,less active, reluctant to feed, abdominal distension, hypotonia, poor reflexes) were enrolled in this study.Neonates with major congenital anomalies,dysmorphism, severe jaundice due to blood group incompatibilities, respiratory distress syndrome requiring respiratory support were excluded from this study. All data were recorded in a specially designed questionnaire form. Written informed consent from the parents of the neonates were taken. Ethical clearance was also taken from the ethical committee of Diabetic Association of Bangladesh. Detailed maternal history was taken and a full physical examination of the babies were done and recorded. Using all aseptic precautions 4-5 $\mathrm{ml}$ of venous blood was taken from each neonate for sepsis work up. Sepsis work up included total white blood cell count, absolute neutrophil count, platelet count, $\mathrm{CRP}$ and Blood $\mathrm{C} / \mathrm{S}$ with antibiotic sensitivity pattern. The reference values of the neonatal haematological parameters of Manroe et $\mathrm{al}^{10}$ and Zeeshan et $\mathrm{al}^{11}$ was used as the standard values (Table 1). A neonate having thrombocytosis or thrombocytopenia was given a score of 1, leukocytosis or leukopenia was given 1, neutrophilia or neutropenia was given 1 and positive CRP was given1 making the total score 4. All the reports were recorded in the data collection sheet. Comparisons were made between the blood culture results and the haematological scores. All the data were processed and analyzed by using SPSS Programme, version 16.0. Data was expressed as mean \pm standard deviation. Comparisons of the variables and the two groups that is the blood culture reports and the haematological scores was done by Chi square test and Fisher's exact test. pd" 0.05 was considered statistically significant.

Table I. Haematological scores used in this study ${ }^{12}$

\begin{tabular}{llc}
\hline Criteria & Abnormality & Score \\
\hline Total WBC & increased or decreased & 1 \\
& $(<5000 / \mathrm{cmm}$ or $\geq 25000 / \mathrm{cmm})$ & \\
ANC & increased or decreased & 1 \\
& $(<2000-2500 / \mathrm{cmm}$ or & \\
& $>7500-8000 / \mathrm{cmm})$ & 1
\end{tabular}

Platelet Count decreased

\begin{tabular}{lll} 
& $\leq 100,000 / \mathrm{cmm})$ & 1 \\
CRP & Positive $(>6 \mathrm{mg} / \mathrm{l})$ & 1 \\
\hline & Total Score & $\mathbf{4}$ \\
\hline
\end{tabular}

Normal values as defined by reference ranges of Monroe et $\mathrm{al}^{10,11}, \mathrm{CRP}=\mathrm{C}$ reactive protein $\mathrm{ANC}=$ Absolute neutrophil count

\section{Results}

The mean age of the 120 neonates was $10 \pm 5.5$ days. Thirty (25\%) neonates had normal birth weight, 64 (53.3\%) neonates had low birth weight and 26 (21.7\%) babies had very low birth weight.Out of 120 neonates, 94 (78.3\%) were preterm ( $<37$ completed weeks). Sixty $(50.8 \%)$ neonates had positive blood culture and 59 $(49.2 \%)$ had negative blood culture reports. Non albicans candida (55.7\%) was found to be the leading organism followed by Acinetobacter (24.6\%), Klebsiella (16.4\%) and Pseudomonas (3.3\%).

Thirty five (29.2\%) neonates had leukopenia, 36 (30 $\%)$ had neutropenia and $80(66.7 \%)$ neonates suffered from thrombocytopenia. CRP was raised in $66(55 \%)$ neonates, $22(18.3 \%)$ had leukocytosis and neutrophilia was present in $10(8.3 \%)$ neonates. Comparisons were made between these haematological values and positive blood culture reports. Leukopenia and neutropenia were found to be statistically significant with positive blood culture reports (Table II). 
Table II. Comparison between haematological parameters and blood culture

\begin{tabular}{lccc}
\hline & \multicolumn{2}{c}{ Blood CS } & \\
\cline { 2 - 3 } & $\begin{array}{c}\text { Positive } \\
\text { Number (\%) }\end{array}$ & $\begin{array}{c}\text { Negative } \\
\text { Number (\%) }\end{array}$ & value \\
\hline Leukopenia & $27(44.3)$ & $8(13.6)$ & 0.001 \\
Neutropenia & $30(49.2)$ & $6(10.2)$ & 0.001 \\
Thrombocytopenia & $42(68.9)$ & $38(64.4)$ & 0.606 \\
Raised CRP & $37(60.7)$ & $29(49.2)$ & 0.205 \\
Leukocytosis & $11(18.0)$ & $11(18.6)$ & 0.931 \\
Neutrophilia & $6(9.8)$ & $4(6.8)$ & 0.743 \\
\hline
\end{tabular}

In this study, regarding the haematological scores, forty two (35\%) neonates had a score of 1 and score 2 was present in forty five (37.5\%) patients. Twenty three (19.2\%) neonates had a score of 3 and 10 neonates $(8.3 \%)$ had a score of 4 . These scores were compared with positive blood culture reports (Table 3 ) and found that $p$ value was significant among the neonates having a score of $1(p=0.001)$. Among the 42 neonates having a score of 1 , culture was negative in 29 of them and only 13 had positive culture reports. Therefore, the chance that those neonates with a score of 1 having sepsis was significantly lower or very unlikely. In the group where the score was 2 , the $\mathrm{p}$ value was not significant (0.479). It was seen that in this group of 45 neonates, almost half had a positive blood culture (21) and the rest were culture negative (24).So it cannot be said with certainty that whether the neonates with a score of 2 will have sepsis or not. $P$ value was significant with scores of 3 and 4 in the present study. Twenty three neonates had a score of 3 with a $p$ value of 0.014 and among the 23 newborns, 17 were proven sepsis by positive culture reports. Blood culture was positive in all the 10 patients with a score of 4 and the $p$ value in this group was 0.001 making this group most susceptible to sepsis. So the interpretation of the scores was naonates having a score of 1 were very unlikely to be septic, those having a score of 2 may or may not suffer from sepsis and neonates having a score of 3 or more were very likely to be septic.
Table III. Comparison of Haematological Score by blood culture and sensitivity

\begin{tabular}{lccc}
\hline Score & \multicolumn{2}{c}{ Blood CS } & $\mathrm{p}$ \\
\cline { 2 - 3 } & $\begin{array}{c}\text { Positive } \\
\text { Number (\%) }\end{array}$ & $\begin{array}{c}\text { Negative } \\
\text { Number (\%) }\end{array}$ & \\
\hline Score 1 & $13(30.9)$ & $29(69.1)$ & 0.001 \\
Score 2 & $21(46.7)$ & $24(53.3)$ & 0.479 \\
Score 3 & $17(74)$ & $6(26)$ & 0.014 \\
Score 4 & $10(100)$ & $0(.0)$ & 0.001 \\
\hline
\end{tabular}

\section{Discussion}

In the current study, almost half of the newborn were diagnosed as culture proven sepsis due to early arrival in hospital, sample collection before giving antibiotic and proper aseptic technique in collection procedure. Among the 120 neonates, 94 (78.3\%) neonates were preterm and two thirds had low birth weight. Mannan et $\mathrm{al}^{13}$ showed that $71.6 \%$ babies developed septicaemia whose birth weight was less than 2500 gram and 79.6 $\%$ babies were preterm. Preterm and very low birth weight babies are susceptible to infections due to low level of $\operatorname{IgG}$ and lower defense mechanism.

Bacterial growth was found in $44.3 \%$ newborns with sepsis in this study. Among the bacteria the commonest organism was acinetobacter $(24.6 \%)$ followed by klebsiella (16.40\%) and pseudomonas (3.30\%). Mishra A found acinetobacter was responsible for neonatal sepsis in $31 \%$ babies $^{14}$ but this study showed that the leading organism was non albicans candida which was isolated in $55.7 \%$ cases. Similar studies by Raha BK also found the most prevalent pathogen to be Non Albicans Candida. ${ }^{15}$ As because most of the newborns were preterm low birth weight, they were exposed to broad-spectrum antibiotics, especially third generation cephalosporins.

The present study showed that, 35 neonates $(29.2 \%)$ had leukopenia, 36 (30\%) had neutropenia and 80 (66.7\%) neonates had thrombocytopenia. Raised CRP was found in $66(55 \%)$ neonates. Leukocytosis was present in $22(18.3 \%)$ patients and $10(8.3 \%)$ had neutrophilia. There was no significant differences between the haematological parameters and blood culture results except leukopenia and neutropenia. This result was consistent with a study by M M Hoque. ${ }^{16}$ Neutropenia has been more common in association with 
sepsis probably because of increased adherence to altered endothelial cells and utilization at the site of infection. ${ }^{4}$ Although the percentage of thrombocytopenia and raised CRP was high in this study there was no significant differences between the culture positive and culture negative groups. Neonates with sepsis developed thrombocytopenia because of disseminated intravascular coagulation and the damaging effect of endotoxin on platelets. . Rodwell $\mathrm{RL}$, Tudehope DL in a study showed that the frequency of thrombocytopenia did not differ significantly between the infected and non infected groups of neutropenic neonates. ${ }^{17}$ Zeeshan et al have also shown poor predictive value for CRP in their study ${ }^{11}$. In our study it was seen that in those neonates with a score of $\geq 3$, the chance of having sepsis was significantly higher. Makkar $\mathrm{M}$ et al also implied that the higher the score the greater the chance of sepsis ${ }^{18}$. Rodwell RL, Leslie AL et al concluded in their study that the higher the score the greater was the likelihood of sepsis and with score less than or equal to 2 the chance of sepsis was very low ${ }^{17}$ making the haematological scoring system a reliable predictor of neonatal sepsis. In a study by Samiksha et al it was concluded that the Haematological scoring system provides an effective guideline to make decisions regarding judicious use of antibiotics, which will be life saving, provide early cure and reduce mortality. ${ }^{19}$

\section{Conclusion}

Neonatal sepsis is a major cause of neonatal mortality and morbidity throughout the world and as well as in Bangladesh. Early diagnosis of neonatal sepsis and appropriate treatment decreases the mortality and morbidity of these newborn. Although we know that blood culture is the gold standard for confirming neonatal sepsis, from this study it can be concluded that a haematological scoring system is useful for early diagnosis of sepsis. In our study, the scoring system implies that the higher the score ( 3 or more) the greater the chance of the neonate having sepsis. Hence the Haematological scoring system plays an important role in differentiating the infected neonates from the non infected ones. It helps the clinicians to make an early diagnosis and start treatment before getting the blood culture reports without further compromising the health of the neonates.

\section{Conflict of interest: None}

\section{References}

1. Zaidi AK, Huskins WC, Thaver D. Hospital-acquired neonatal infections in developing countries. Lancet. 2005;365:117588.

2. Bangladesh Demographic and Health Survey 2014. 102 p.

3. Ahmed AS, Chowdhury MA, Hoque M. Clinical and Bacteriological profile of Neonatal Septicaemia in Tertiary Level Pediatric Hospital in Bangladesh. Indian Pediatrics 2002; 39 (11) : 1034-39

4. Berger C, Uehlinger J, Ghelfi D. Comparison of C reactive protein and white blood cell count with differential in neonates at risk of septicaemia. Eur J Pediatr 1995; 154: 138-44

5. Kawamura M, Nishida $H$. The usefulness of serial $\mathrm{C}$ reactive protein measurement in managing neonatal infection. Acta Paediatr 1995;84:10-13.

6. Gomella TL, Cunningham MD, Eyal FG et al. Neonatology: management, procedures, on call problems, diseases, and drugs. $5^{\text {th }}$ edn. New York: McGraw Hill; 2004. Pp. 77-101.

7. Peter D. Infection in the newborn. In: Rennie JM. Robertson's NRC. Textbook of Neonatology. $4^{\text {th }}$ ed. London Elsevier Churchill Livingstone 2005; 1017 - 18.

8. Modanlou HD, Ortiz OB. Thrombocytopenia in neonatal infection. Clin pediatr (phila) 1981; 20: 402-07.

9. Storm W. Use of thrombocytopenia for the early identifications of sepsis in critically ill newborns. Acta paediatr Acad Sci Hung 1982; 23: 349-55.

10. Monroe BL, Weinberg AG, Rosenfeld CR. The neonatal blood count in health and disease. I. Reference values for neutrophilic cells. J Pediatr 1979;95:89-98

11. Ahmed Z, Ghafoor T, Walker T. Diagnostic value of C reactive protein and haematological parameters in neonatal sepsis. $J$ Coll Physicians Surg Pak 2005 Mar; 15(3):152-56

12. Shirazi H, Riaz S, Tahir R. Role of Haematological Profile in Early Diagnosis of Neonatal Sepsis. Ann Pak Inst Med Sci 2010; 6(3):153.

13. Mannan MA, Shahidullah M, Noor MK. In utility of Creactive protein and heamatological parameters in the detection of neonatal sepsis. Bangladesh Journal of Child Health 2005;45:64-69.

14. Mishra A, Mishra S, Jaganath Gl. Acinatobacter Sepsis in Newborns. Indian Pediatrics 1998;35:27-33.

15. Raha BK, Baki MA, Begum T. Organism specific response of platelet count in neonatal sepsis. BIRDEM Med J 2014; 4(2): 79-83.

16. MM Hoque, ASMNU Ahmed, SK Halder et al. Clinical manifestation and bacteriological profile of septicaemia in preterm neonates. Bangladesh J Med Sci 2004; 10 (1): 29-33

17. Rodwell RL, Tudehope DI. Haematological scoring system in early diagnosis of sepsis in neutropenic newborns. Pediatr Infect Dis $J$ 1993; 12: 372-76.

18. Makkar M, Gupta C, Pathak R. Performance Evaluation of Haematologic Scoring System in Early Diagnosis of Neonatal Sepsis. J Clin Neonatol 2013; 2(1): 25-29.

19. Ablawat S, Chand N, Mehta S et al. Assessment of Haematological Scoring System in Early Diagnosis of Neonatal Sepsis in Term and Pre Term Neonates. ejpmr 2017;4(3):390 (online). 